ANNALES

POLONICI MATHEMATICI

$82.1(2003)$

\title{
Multiplicative Cauchy functional equation in the cone of positive-definite symmetric matrices
}

\author{
by Konstancja Bobecka and Jacek WesoŁowski (Warszawa)
}

\begin{abstract}
We solve the multiplicative Cauchy equation for real functions of symmetric positive definite matrices under the differentiability restriction. The specialty of the problem lies in the symmetry of the multiplication.
\end{abstract}

1. Introduction. The multiplicative Cauchy equation in the space of real square matrices of the form

$$
f(x y)=f(x) f(y),
$$

where $x, y$ are matrices and $f$ is a real-valued function, was studied intensively in late fifties and the beginning of sixties, with the main contributions by Gołąb (1959), Hosszú (1959), Kucharzewski and Zajtz (1966), Kuczma (1959), Djoković (1970). It is known that the general solution is of the form

$$
f(x)=g(\operatorname{det}(x)),
$$

where $g$ is a multiplicative function, and if additionally some smoothness assumptions are imposed on $f$ the solution is $f(x)=|\operatorname{det}(x)|^{\lambda}$, where $\lambda$ is a real number. A related Cauchy-Binet equation (having the same solution) has been studied for instance in Kurepa (1964), and more recently in Heuvers, Cummings and Bhaskara-Rao (1988) and Heuvers and Moak (1990, 1991).

Our interest in functional equations in the cone of positive-definite symmetric matrices was motivated by investigations of characterization problems for probabilistic measures concentrated on positive-definite symmetric matrices - see Letac and Wesołowski (2000), Bobecka and Wesołowski (2002) and Wesolowski (2002) and the equations treated there. To our surprise nothing has been done for the Cauchy equation in this cone. This note, at least to the best of our knowledge, is the first attempt in this direction.

2000 Mathematics Subject Classification: 39B52, 15A15, 39B22.

Key words and phrases: symmetric positive-definite matrices, determinant, multiplicative Cauchy functional equation, symmetric multiplication. 
2. Preliminaries and the derivative of the determinant. Let $\mathcal{V}_{+}$ denote the cone of positive-definite symmetric real $n \times n$ matrices. It is a subset of the Euclidean space $\mathcal{V}$ of symmetric real $n \times n$ matrices endowed with the inner product $(a, b)=\operatorname{tr}\left(a b^{\mathrm{T}}\right)=\operatorname{tr}(a b)$ for any $a, b \in \mathcal{V}$, where $a^{\mathrm{T}}$ denotes the transpose and $\operatorname{tr}(a)$ denotes the trace of the matrix $a$.

We are interested here in a version of the multiplicative Cauchy functional equation for an unknown function $f: \mathcal{V}_{+} \rightarrow(0, \infty)$. The natural version of the Cauchy equation in $\mathcal{V}_{+}$has the form

$$
f(x) f(y)=f\left(y^{1 / 2} x y^{1 / 2}\right)
$$

for any $x, y \in \mathcal{V}_{+}$, i.e. the multiplication in the argument of $f$ on the right hand side of (1) is symmetric. Obviously, the function $f(x)=(\operatorname{det} x)^{\lambda}$ for any real $\lambda$ is a solution of (1). It will be shown that this is the only possible solution in the class of differentiable functions $f$.

First we introduce some linear operators which will be used in the course of the proof of our main result.

For any $y \in \mathcal{V}_{+}$let us define two endomorphisms $\mathbb{P}(y)$ and $\mathbb{L}(y)$ on $\mathcal{V}$ :

$$
\mathbb{P}(y) h=y h y, \quad \mathbb{L}(y) h=y h+h y, \quad h \in \mathcal{V} .
$$

Observe that the inverse operator to $\mathbb{P}(y)$, denoted by $\mathbb{P}^{-1}(y)$, exists and $\mathbb{P}(y)^{-1}=\mathbb{P}\left(y^{-1}\right)$. Also the inverse $\mathbb{L}^{-1}(y)$ of $\mathbb{L}(y)$ exists- see for instance Olkin and Rubin (1964). In what follows we will use two simple relations involving both these operators:

$$
\mathbb{L}\left(y^{2}\right) \mathbb{P}\left(y^{-1}\right)=\mathbb{P}\left(y^{-1}\right) \mathbb{L}\left(y^{2}\right)
$$

and

$$
\mathbb{L}^{2}(y)=\mathbb{L}\left(y^{2}\right)+2 \mathbb{P}(y)
$$

for any $y \in \mathcal{V}_{+}$.

Before we present the solution of equation (1) we give an auxiliary result which seems to be of independent interest:

Proposition 1. Assume that for any $y \in \mathcal{V}_{+}$,

$$
\mathbb{L}\left(y^{2}\right) b=2 \mathbb{P}(y) b
$$

for some $b \in \mathcal{V}$. Then there exists $\lambda \in \mathbb{R}$ such that $b=\lambda e$, where e denotes the identity matrix in $\mathcal{V}$.

Proof. By (4) we get

$$
\begin{aligned}
0 & =\left(\mathbb{L}\left(y^{2}\right) b-2 \mathbb{P}(y) b, b\right)=\left(y^{2} b, b\right)-(y b y, b)+\left(b y^{2}, b\right)-(y b y, b) \\
& =(b y, y b)-(y b, y b)+(b y, y b)-(b y, b y) \\
& =(b y-y b, y b)+(b y, y b-b y)=(b y-y b, y b)-(b y-y b, b y) \\
& =(b y-y b, y b-b y)=\left(b y-y b,(b y-y b)^{\mathrm{T}}\right)=\|b y-y b\|^{2},
\end{aligned}
$$

where $\|\cdot\|$ denotes the norm generated by the inner product. 
Then for any $y \in \mathcal{V}_{+}$it follows that $b y=y b$. Insert in this identity $y=y_{i j}=e+\varepsilon e_{i j}$ for any $i, j \in\{1, \ldots, n\}$, where $e_{i j}$ is an $n \times n$ matrix with zero entries, except the $(i, j)$ and $(j, i)$ entries which are 1 , and $\varepsilon$ is chosen sufficiently small to have $y_{i j} \in \mathcal{V}_{+}$. Then, if $b=\left[b_{i j}\right]$, it follows that $b_{i k}=0$ for any $k \neq i$ and $b_{i i}=b_{j j}$ for any $i, j=1, \ldots, n$. Thus $b=\lambda e$ for some real $\lambda$.

We end this section with the result on the derivative of the determinant function, which is known in mathematical folklore, but we do not know of any exact reference. Consequently, to make the paper self-contained, we offer a fairly transparent proof.

Proposition 2. Consider a determinant function det on the cone $\mathcal{V}_{+}$. Then

$$
(\operatorname{det} x)^{\prime}=(\operatorname{det} x) x^{-1}, \quad x \in \mathcal{V}_{+} .
$$

Proof. For any $x \in \mathcal{V}_{+}$and $h \in \mathcal{V}$ such that $x+h \in \mathcal{V}_{+}$we have

$$
\begin{aligned}
\operatorname{det}(x+h)-\operatorname{det}(x) & =\operatorname{det}\left(x^{1 / 2}\left(e+x^{-1 / 2} h x^{-1 / 2}\right) x^{1 / 2}\right)-\operatorname{det}(x) \\
& =\operatorname{det}(x)\left[\operatorname{det}\left(e+x^{-1 / 2} h x^{-1 / 2}\right)-1\right] .
\end{aligned}
$$

Observe now that for any $a \in \mathcal{V}_{+}$one has

$$
\operatorname{det}(e+a)=1+\sum_{k=1}^{n} \sum_{\left\{i_{1}, \ldots, i_{k}\right\} \subset\{1, \ldots, n\}} A_{i_{1}, \ldots . i_{k}},
$$

where $A_{i_{1}, \ldots, i_{k}}$ denotes the principal minor based on rows and columns of indices $i_{1}, \ldots, i_{k}$. Note that the first element of the above sum is just $\operatorname{tr}(a)$. Further, for any minor of order $k \geq 2$ we have

$$
\left|A_{i_{1}, \ldots, i_{k}}\right| \leq k !\left(\max _{i, j}\left|a_{i j}\right|\right)^{k}
$$

But

$$
\left(\max _{i, j}\left|a_{i j}\right|\right)^{k} \leq\left(\sqrt{\sum_{i, j} a_{i j}^{2}}\right)^{k}=\|a\|^{k} .
$$

Consequently,

$$
\frac{\left|A_{i_{1}, \ldots, i_{k}}\right|}{\|a\|} \leq k !\|a\|^{k-1},
$$

and since (for $k \geq 0$ ) the right hand side tends to zero as $\|a\| \rightarrow 0$ we have

$$
\lim _{\|a\| \rightarrow 0} \frac{|\operatorname{det}(e+a)-1-\operatorname{tr}(a)|}{\|a\|}=0 \text {. }
$$

Now take $a=a(h)=x^{-1 / 2} h x^{-1 / 2}$ and note that $\|a(h)\| \leq\left\|x^{-1 / 2}\right\|^{2}\|h\|$ $\rightarrow 0$ as $\|h\| \rightarrow 0$. Since $\operatorname{tr}\left(x^{-1 / 2} h x^{-1 / 2}\right)=\operatorname{tr}\left(x^{-1} h\right)$, we have 


$$
\begin{aligned}
& \frac{\left|\operatorname{det}(x+h)-\operatorname{det}(x)-\operatorname{det}(x) \operatorname{tr}\left(x^{-1} h\right)\right|}{\|h\|} \\
& \quad=\operatorname{det}(x) \frac{\left|\operatorname{det}\left(e+x^{-1 / 2} h x^{-1 / 2}\right)-1-\operatorname{tr}\left(x^{-1} h\right)\right|}{\left\|x^{-1 / 2} h x^{-1 / 2}\right\|} \frac{\left\|x^{-1 / 2} h x^{-1 / 2}\right\|}{\|h\|} \\
& \quad \leq \operatorname{det}(x) \frac{\left|\operatorname{det}\left(e+x^{-1 / 2} h x^{-1 / 2}\right)-1-\operatorname{tr}\left(x^{-1} h\right)\right|}{\left\|x^{-1 / 2} h x^{-1 / 2}\right\|}\left\|x^{-1 / 2}\right\|^{2}
\end{aligned}
$$

since $\left\|x^{-1 / 2} h x^{-1 / 2}\right\| \leq\left\|x^{-1 / 2}\right\|^{2}\|h\|$. Finally, (6) implies that

$$
\lim _{\|h\| \rightarrow 0} \frac{\left|\operatorname{det}(x+h)-\operatorname{det}(x)-\operatorname{det}(x) \operatorname{tr}\left(x^{-1} h\right)\right|}{\|h\|}=0,
$$

and thus (5) is proved.

3. Cauchy equation. Now we are ready to state and prove our main result.

Theorem 1. Let the function $f: \mathcal{V}_{+} \rightarrow(0, \infty)$ be differentiable. If $f$ satisfies equation (1) then there exists $\lambda \in \mathbb{R}$ such that $f(x)=(\operatorname{det} x)^{\lambda}$ for any $x \in \mathcal{V}_{+}$.

Proof. Let $g=\log f$. Then (1) can be rewritten as

$$
g\left(y^{1 / 2} x y^{1 / 2}\right)=g(x)+g(y), \quad x, y \in \mathcal{V}_{+} .
$$

For $y$ changed to $y^{2}$ we get

$$
g(\mathbb{P}(y) x)=g(x)+g\left(y^{2}\right), \quad x, y \in \mathcal{V}_{+} .
$$

Differentiate (7) with respect to $x$ to get

$$
\mathbb{P}(y) g^{\prime}(\mathbb{P}(y) x)=g^{\prime}(x) .
$$

Since $\mathbb{P}(y) e=y^{2}$, plugging $x=e$ in (8), we obtain

$$
g^{\prime}\left(y^{2}\right)=\mathbb{P}\left(y^{-1}\right) g^{\prime}(e) .
$$

Set $b=g^{\prime}(e) \in \mathcal{V}_{+}$and substitute back $y$ for $y^{2}$. Then

$$
g^{\prime}(y)=\mathbb{P}\left(y^{-1 / 2}\right) b, \quad y \in \mathcal{V}_{+} .
$$

Now we differentiate (7) with respect to $y$. Then it follows that

$$
x y g^{\prime}(\mathbb{P}(y) x)+g^{\prime}(\mathbb{P}(y) x) y x=\mathbb{L}(y) g^{\prime}\left(y^{2}\right) .
$$

In the above equation insert $y=e$. Since $\mathbb{P}(e) x=x$, (9) implies

$$
x g^{\prime}(x)+g^{\prime}(x) x=\mathbb{L}(x) g^{\prime}(x)=\mathbb{L}(e) g^{\prime}\left(e^{2}\right)=2 b .
$$


Consequently, by (9) it follows that

$$
\mathbb{L}(x) \mathbb{P}\left(x^{-1 / 2}\right) b=2 b,
$$

which, upon changing $x$ to $x^{2}$ and then applying (2), can be written as

$$
\mathbb{P}\left(x^{-1}\right) \mathbb{L}\left(x^{2}\right) b=2 b .
$$

Finally we get

$$
\mathbb{L}\left(x^{2}\right) b=2 \mathbb{P}(x) b, \quad x \in \mathcal{V}_{+} .
$$

Hence, by Proposition 1 , it follows that $b=\lambda e$ for some $\lambda \in \mathbb{R}$. And thus (9) implies $g^{\prime}(x)=\lambda x^{-1}, x \in \mathcal{V}_{+}$, which via Proposition 2 leads to $g(x)=\lambda \log \operatorname{det} x+c$, where $c$ is a real constant. Then $f(x)=e^{c}(\operatorname{det} x)^{\lambda}$ and it follows by (1) that $c=0$, which ends the proof.

The symmetric product of symmetric positive-definite matrices $x$ and $y$ defined as $y^{1 / 2} x y^{1 / 2}$ may look somewhat arbitrary, since other candidates for products preserving symmetry and positive definiteness are possible. The one we consider seems to be quite natural, at least in the context of characterizations of probability measures on the cone $\mathcal{V}_{+}$. Another natural choice of the symmetric product of $x$ and $y$ is

$$
\frac{x y+y x}{2}=\frac{1}{2} \mathbb{L}(x) y,
$$

which is known as the Jordan product in the Jordan algebra of symmetric matrices (see Faraut and Korányi (1994)). In this case the multiplicative Cauchy equation reads

$$
f(x) f(y)=f\left(\frac{x y+y x}{2}\right), \quad x, y \in \mathcal{V}_{+} .
$$

It turns out that this equation admits essentially only trivial solutions.

Theorem 2. Let the function $f: \mathcal{V}_{+} \rightarrow(0, \infty)$ be differentiable. If $f$ satisfies (10) then $f=1$.

Proof. As above we introduce $g=\log f$. Then (10) can be written as

$$
g(x)+g(y)=g\left(\frac{1}{2} \mathbb{L}(x) y\right) .
$$

Upon differentiation with respect to $x$ we get

$$
g^{\prime}(x)=\frac{1}{2} \mathbb{L}(y) g^{\prime}\left(\frac{1}{2} \mathbb{L}(x) y\right) .
$$

Hence plugging $x=e$ in (11) and writing $b=g^{\prime}(e)$ we obtain

$$
2 b=\mathbb{L}(y) g^{\prime}\left(y^{2}\right) .
$$


On the other hand inserting $x=y$ in (11) we arrive at

$$
2 g^{\prime}(x)=\mathbb{L}(x) g^{\prime}\left(x^{2}\right) .
$$

Consequently,

$$
2 \mathbb{L}^{-1}(x) b=\mathbb{L}(x) \mathbb{L}^{-1}\left(x^{2}\right) b .
$$

Applying the operator $\mathbb{L}(x)$ to both sides and then using (3) we find that

$$
b=2 \mathbb{P}(x) \mathbb{L}^{-1}\left(x^{2}\right) b .
$$

Finally we use (2) and then apply $\mathbb{L}\left(x^{2}\right)$ to both sides, arriving at (4). Now repeating the argument from the proof above (using Proposition 1) we find that the solution of (10) has to be of the form $f(x)=[\operatorname{det}(x)]^{\lambda}$, where $\lambda \in \mathbb{R}$. Putting it back into (10) yields $\lambda=0$.

Acknowledgements. The presentation of the material has been considerably improved thanks to the referee's suggestions. Particularly his/her remark on symmetric products motivated us to incorporate Theorem 2 in the paper.

\section{References}

K. Bobecka and J. Wesołowski (2002), The Lukacs-Olkin-Rubin theorem without invariance of the "quotient", Studia Math. 152, 147-160.

D. Ž. Djoković (1970), On homomorphisms of the general linear group, Aequationes Math. 4, 99-102.

J. Faraut and A. Korányi (1994), Analysis on Symmetric Cones, Oxford Univ. Press, New York.

S. Gołąb (1959), Sur l'équation $f(X) f(Y)=f(X Y)$, Ann. Polon. Math. 6, 1-13.

K. J. Heuvers, L. J. Cummings and K. P. S. Bhaskara-Rao (1988), A characterization of the permanent function by the Binet-Cauchy theorem, Linear Algebra Appl. 101, 49-72.

K. J. Heuvers and D. S. Moak (1990), The Binet-Pexider functional equation for rectangular matrices, Aequationes Math. 40, 136-146.

K. J. Heuvers and D. S. Moak (1991), The solution of the Binet-Cauchy functional equation for square matrices, Discrete Math. 88, 21-32.

M. Hosszú (1959), A remark on scalar valued multiplicative functions of matrices, Publ. Math. Debrecen 6, 288-289.

M. Kucharzewski und A. Zajtz (1966), Über die linearen homogenen geometrischen Objekte des Typus $[m, n, 1]$, wo $m \leq n$ ist, Ann. Polon. Math. 18, 205-225.

M. Kuczma (1959), Bemerkung zur vorhergehenden Arbeit von M. Kucharzewski, Publ. Math. Debrecen 6, 199-203.

S. Kurepa (1964), On the characterization of the determinant, Glas. Mat.-Fiz. Astronom. Ser. II 19, 189-198.

G. Letac and H. Massam (1998), Quadratic and inverse regressions for Wishart distributions, Ann. Statist. 26, 573-595.

G. Letac and J. Wesołowski (2000), An independence property for the product of GIG and gamma laws, Ann. Probab. 28, 1371-1383. 
I. Olkin and H. Rubin (1964), Multivariate beta distributions and independence properties of the Wishart distribution, Ann. Math. Statist. 35, 261-269.

J. Wesołowski (2002), The Matsumoto-Yor independence property for GIG and gamma laws, revisited, Math. Proc. Cambridge Philos. Soc. 133, 153-161.

Faculty of Mathematics and Information Science

Warsaw University of Technology

00-661 Warszawa, Poland

E-mail: bobecka@mini.pw.edu.pl

wesolo@mini.pw.edu.pl

Reçu par la Rédaction le 4.5.2001

Révisé le 9.10.2002 